\title{
COMPETENCIA DE LeCheRON (Euphorbia dentata) EN SOJA ${ }^{1}$
}

\author{
Competition of Spurge Toothed (Euphorbia dentata) in Soybean
}

JUAN, V.F. ${ }^{2}$, SAINT-ANDRE, H. ${ }^{2}$ y FERNANDEZ, R.R. ${ }^{3}$

\begin{abstract}
RESUMEN - Euphorbia dentata es una maleza anual, de ciclo primavero - estival que está presente en el $85 \%$ del área agrícola del partido de Azul, Pcia Buenos Aires Argentina, con densidades que oscilan entre 20 a 200 plantas $\mathrm{m}^{-2}$. Estudios en campo fueron conducidos durante dos años para determinar la relación de competencia entre soja y E. dentata a través de la evaluación del efecto de distintas densidades de la maleza sobre los componentes del rendimiento del cultivo y su biomasa. El peso seco y rendimiento de soja mostraron una respuesta dependiente de la densidad y de la biomasa de la maleza, siendo reducidos al $80 \%$ por la interferencia a partir de la densidad de 20 plantas $\mathrm{m}^{-2}$. En el rango de densidades estudiadas se observó una respuesta lineal que fue ajustada a una ecuación de regresión que indica que la producción de semillas de soja decrece $6,73 \mathrm{~kg} \mathrm{ha}^{-1}$ por el incremento de cada planta $\mathrm{m}^{-2}$ de E. dentata. De los componentes del rendimiento del cultivo, el número de vainas por planta fue el más severamente afectado por la competencia obteniéndose reducciones del $40 \%$ respecto del tratamiento sin maleza. Altos niveles de correlación fueron observados entre el rendimiento de soja, el número de vainas por planta y el peso de 1.000 semillas (98 y $85 \%$ respectivamente).
\end{abstract}

Palabras claves: rendimiento, densidad, biomasa, Glycine max, EPHDE.

ABSTRACT - Euphorbia dentata is a spring-summer annual weed first detected in Azul Co., Buenos Aires Province, Argentina., in 1983, in an isolated soybean crop spot crops. It is now found as a weed in $85 \%$ of the cropland, with densities ranging from 20 to 200 plants $\mathrm{m}^{2}$. Two year- field studies were conducted to determine the competition relationship between soybean and $\boldsymbol{E}$. dentata. The effect of different weed densities was evaluated on yield parameters and biomass. Both soybean dry weight and yield gave a significant response to weed density and biomass, resulting in a reduction of $80 \%$ due to interference starting at a density of 20 plants $\mathrm{m}^{2}$. It was determined that $\boldsymbol{E}$. dentata has a strong influence on soybean yield showing a linear response at the range of the studied densities, fitted to a regression equation indicating that seed production decreases $6,73 \mathrm{~kg} \mathrm{ha}^{1}$ for each plant $\mathrm{m}^{-2}$ increase of $\boldsymbol{E}$. dentata. From the crop yield components, the number of pods per plant was more severely affected by competition reducing yield in $40 \%$, when compared to the unweeded treatment. The number of grains per pod and weight of 1000 seeds were significantly affected at high densities of $\boldsymbol{E}$. dentata (55 plants $\mathrm{m}^{-2}$ ). High correlation levels were found between soybean seed yield, number of pods per plant and weight of 1000 seeds (98 and $85 \%$ respectively).

Key words: crop yield, weed densities, biomass, Glycine max, EPHDE.

Recebido para publicação em 4.10.2002 e na forma revisada em 11.8.2003.

Ingeniero Agrónomo, Profesor Adjunto Cátedra de Terapéutica Vegetal, Facultad de Agronomía Universidad Nacional del Centro de la Provincia de Buenos Aires, Casilla de Correo 47, (7300) - Azul, Buenos Aires, Argentina. <vjuan@faa.unicen.edu.ar>, $<$ hsaint@faa.unicen.edu.ar>. ${ }^{3}$ Ingeniero Agrónomo, Profesor Asociado Cátedra de Terapéutica Vegetal, Facultad de Agronomía Universidad Nacional del Centro de la Provincia de Buenos Aires, <rrf@faa.unicen.edu.ar>. 


\section{INTRODUCCIÓN}

La competencia entre soja y malezas es un fenómeno complejo que ha sido y es motivo de estudios permanentes debido a la importancia que esta relación tiene sobre los componentes del rendimiento del cultivo y también por que es la base para la elaboración de los programas de control de malezas.

Euphorbia dentata es una especie anual, de ciclo primavero - estival que fue citada por primera vez en la República Argentina por Parodi (1930). En el Partido de Azul, Pcia. de Bs. As., se la ha detectado a partir del año 1983 , localizada en focos aislados en cultivos de soja y actualmente se la encuentra en el $85 \%$ del área agrícola, 250.000 has, en densidades que van desde 20 a 200 plantas $\mathrm{m}^{-2}$ (Juan et al., 1996).

En esta zona $E$. dentata es considerada una especie altamente competitiva y de dificil control debido a la baja eficacia de la mayoría de los tratamientos químicos y la alta dependencia del estado fenológico al momento del control (Juan \& Saint-André, 1997; Juan et al., 2000).

Si bien inicialmente fue caracterizada como maleza en soja, en la actualidad también está presente en cultivos de girasol y maíz, e incluso en trigos de ciclo corto a partir del mes de octubre (Juan et al., 1996).

En la zona sojera central de la República Argentina que abarca centro y sur de la provincia de Santa Fé, norte de la provincia de Buenos Aires y centro y sudeste de la provincia de Córdoba, es una maleza de importancia secundaria, debido a que se encuentra presente sólo en un porcentaje bajo de los lotes, comparativamente con otras especies (Giorda \& Baigorri, 1997). Sin embargo en el área de estudio, su población se encuentra cada vez con mayor frecuencia dominando las comunidades de malezas a pesar de los tratamientos con herbicidas, lo que indica la dificultad que representa su control químico y la consecuente expansión del área ocupada por la misma.

Existen pocos antecedentes sobre E. dentata, pero la bibliografía indica que la especie tiene ciertas características que la asemejan con otra Euforbiácea anual ampliamente difundida como es Euphorbia heterophylla, que es una de la principales malezas latifoliadas en cultivos de soja de diversos países del mundo y que ha sido motivo de numerosos estudios (Nester et al., 1979; Harger \& Nester, 1980; Wilson, 1981; Willard et al., 1994).

El objetivo de este trabajo fue determinar la relación de competencia entre soja y Euphorbia dentata a través de la evaluación del efecto de distintas densidades de la maleza sobre los componentes del rendimiento del cultivo y su biomasa.

\section{MATERIALES Y MÉTODOS}

Ensayos de campo fueron realizados durante las campañas agrícolas 1997/98 y 1998/99 en el partido de Azul, Provincia de Buenos Aires Argentina, en lotes con alta infestación de E. dentata. El área experimental fue sembrada el 10 de noviembre de 1997 y el 13 de noviembre de 1998 con soja cultivar AX 4422, perteneciente al grupo IV, espaciada a $70 \mathrm{~cm}$ entre hileras, en parcelas de $3,5 \mathrm{x}$ $6 \mathrm{~m}$ con una densidad media de 42 plantas $\mathrm{m}^{-2}$. Utilizando experimentos aditivos se establecieron desde la emergencia, mediante raleo las siguientes densidades de E. dentata: 0; 20; 55 ; 90 y 125 plantas $\mathrm{m}^{-2}$, las que se mantuvieron hasta la cosecha. Las otras especies de malezas fueron eliminadas manualmente durante el período del ensayo.

Al finalizar la estación de crecimiento las parcelas fueron cosechadas determinándose peso seco de E. dentata y soja, y los componentes del rendimiento del cultivo: número de vainas por planta, número de semillas por vaina y peso de 1.000 semillas.

El diseño experimental fue de bloques al azar con 5 repeticiones, los datos fueron analizados por análisis de varianza y las medias fueron comparadas por SNK test a nivel de 5\%. Los datos de rendimiento fueron sujetos a análisis de regresión y sus componentes correlacionados entre sí.

\section{RESULTADOS Y DISCUSIÓN}

Tanto el peso seco de soja como el rendimiento mostraron una respuesta significativamente dependiente de la densidad y de la 
biomasa de la maleza, siendo afectados por la interferencia a partir del nivel más bajo de densidad de $E$. dentata ensayado (Figura 1 y 2). En efecto, estas variables fueron reducidas en un $20 \%$ con respecto al tratamiento $\sin$ maleza por la densidad de 20 plantas $\mathrm{m}^{-2}$ (Figura 1). Con esta densidad, las plantas de E. dentata acumularon una cantidad alta de biomasa seca por planta (32 g) que representó aproximadamente $600 \mathrm{~g} \mathrm{~m}^{-2}$ (Figura $2 \mathrm{y} 3$ ).

Con densidades de 55 plantas $\mathrm{m}^{-2}$ el rendimiento de soja fue reducido al $73 \%$ respecto al testigo, resultando además, significativamente menor que el obtenido con 20 planta $\mathrm{m}^{-2}$. Además, en esta situación la mayor intensidad de competencia no fue reflejada en diferencias en la biomasa del cultivo cuyo peso seco no resultó significativamente menor con respecto al tratamiento anterior (Figura 1), pero en la maleza se produjo una reducción significativa en su biomasa por planta $(24 \mathrm{~g})$ y acumuló $1.400 \mathrm{~g} \mathrm{~m}^{-2}$ de peso seco total (Figura 3).

Cuando la densidad de $E$. dentata fue de 90 plantas $\mathrm{m}^{-2}$, la biomasa de soja fue reducida al $72 \%$ y su rendimiento en producción de semillas al 69\%, no detectándose diferencias respecto al tratamiento de 55 plantas $\mathrm{m}^{-2}$. A esta densidad, solo resultó significativa la competencia intraespecífica ya que en la maleza el peso seco se redujo a $19 \mathrm{~g}$ por planta y acumuló $1700 \mathrm{~g} \mathrm{~m}^{-2}$ de biomasa (Figura 3).

Con la máxima densidad, 125 plantas $\mathrm{m}^{-2}$, ambos peso seco y producción de semillas de soja fueron reducidos significativamente un 57 y $55 \%$ respectivamente en relación al tratamiento sin maleza. El peso seco por planta de $E$. dentata no fue afectado, pero en función a la densidad su biomasa alcanzó $2200 \mathrm{~g} \mathrm{~m}^{-2}$ (Figura 2).

E. dentata tuvo una gran influencia sobre el rendimiento de soja mostrando en el rango de densidades estudiadas una respuesta lineal representada por las siguientes ecuaciones de regresión:

$$
\begin{aligned}
& \mathrm{y}_{1}\left(\mathrm{~g} \mathrm{~m}^{-2}\right)=202,46-0,673 \cdot \mathrm{x}_{1} \quad\left(\mathrm{r}^{2}=0,907\right) \\
& \mathrm{y}_{2}\left(\mathrm{~g} \mathrm{~m}^{-2}\right)=206,20-0,0391 \cdot \mathrm{x}_{2} \quad\left(\mathrm{r}^{2}=0,956\right)
\end{aligned}
$$

donde: $\mathrm{y}_{1}$ ó $\mathrm{y}_{2}$ representan el rendimiento calculado en función de la densidad expresada en plantas $\mathrm{m}^{-2}\left(\mathrm{x}_{1}\right)$ ó del peso seco de la maleza expresado en $\mathrm{g} \mathrm{m}^{-2}\left(\mathrm{x}_{2}\right)$ (Figura 1 y 2 ).

En base a los resultados cuantificados, la producción de semillas de soja decrece $6,73 \mathrm{~kg} \mathrm{ha}^{-1}$ por cada planta $\mathrm{m}^{-2}$ que incrementa $E$. dentata ó en forma similar decrece $0,391 \mathrm{~kg} \mathrm{ha}^{-1}$ por cada $1000 \mathrm{~g} \mathrm{~m}^{-2}$ de incremento de peso seco de la maleza, representando estos valores las pendientes de las rectas estimadas.

Dew (1972), desarrolló un modelo lineal de este tipo para estudiar la relación de competencia entre trigo y Avena fatua e introdujo el concepto de indice de competencia determinando la pérdida provocada por distintas densidades de la maleza en este cultivo.

Posteriormente otros índices de competencia para especies individuales de malezas han sido determinados con el objeto de evaluar la habilidad competitiva de las distintas especies que invaden los cultivos, para comparar la respuesta de distintos cultivares y en última instancia para desarrollar modelos de simulación multiespecíficos. Estos últimos permiten predecir las pérdidas ocasionadas por varias especies de malezas creciendo al mismo tiempo en un cultivo y bajo un amplio rango de condiciones e integran los efectos provocados cuando el sistema tiene varios recursos limitantes, diferencias en cuanto al momento de emergencia de las malezas respecto de la emergencia del cultivo, diferentes períodos de convivencia y otros (Coble, 1985; Swanton \& Weise, 1991; Weaver, 1996).

Las mayoría de las ecuaciones que caracterizan las relaciones individuales de competencia entre una maleza y los cultivos están representadas por gráficos de forma hiperbólica (Cousens, 1985), no obstante Zimdahl (1980) describe esta relación a través de una curva sigmoidal y propone que la presencia de malezas en bajas densidades no provoca pérdidas de productividad detectables.

En el presente estudio la relación de competencia entre E. dentata y soja muestra que aún bajas densidades de la maleza determinan disminuciones de rendimiento significativas, y que en estas condiciones las plantas de $E$. dentata acumularon una gran cantidad de biomasa relativa al tamaño lo que ocasionó una menor productividad en el cultivo. 


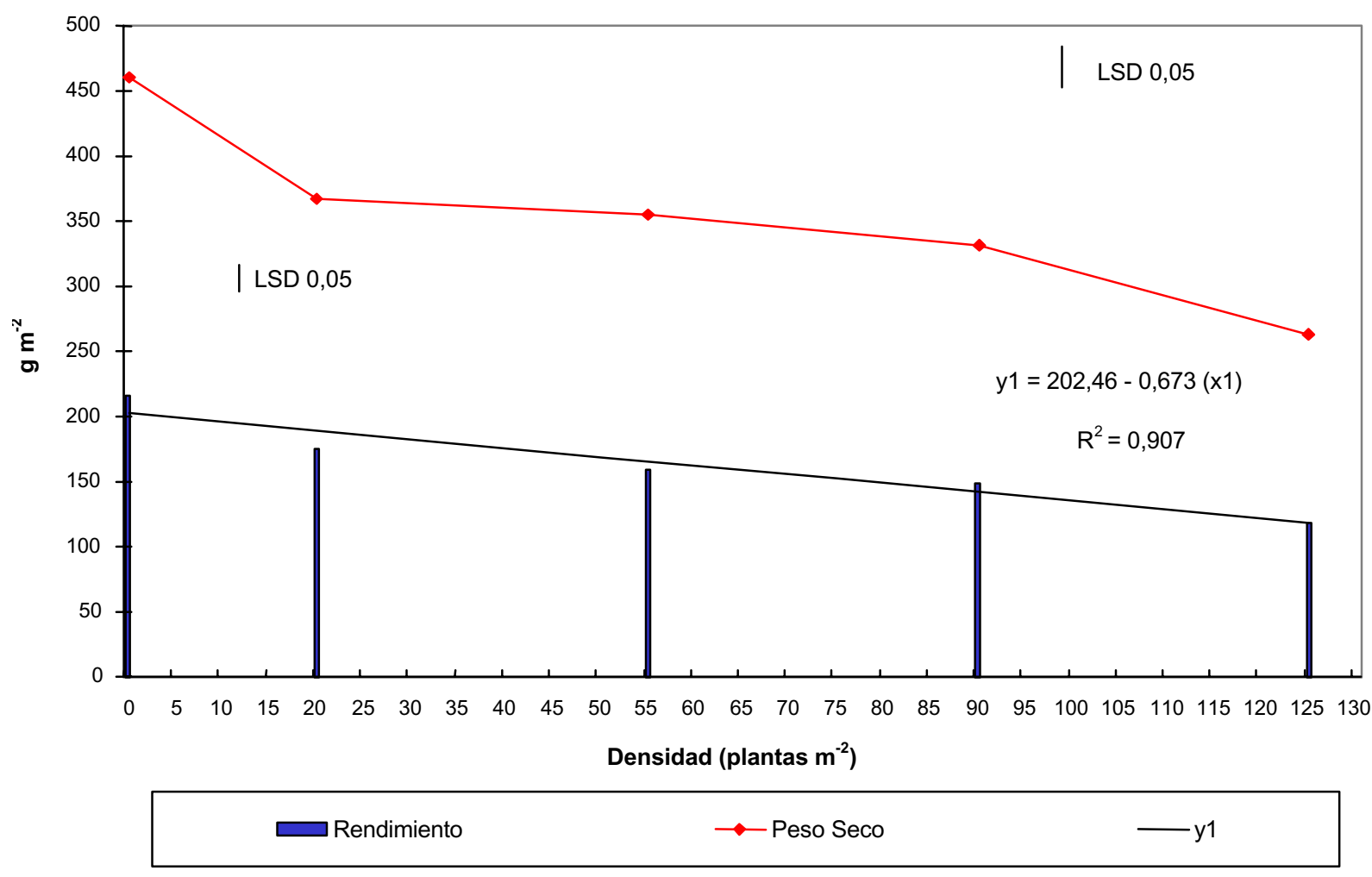

Figura 1 - Efecto de las densidades de Euphorbia dentata sobre la biomasa seca y productividad de soja.

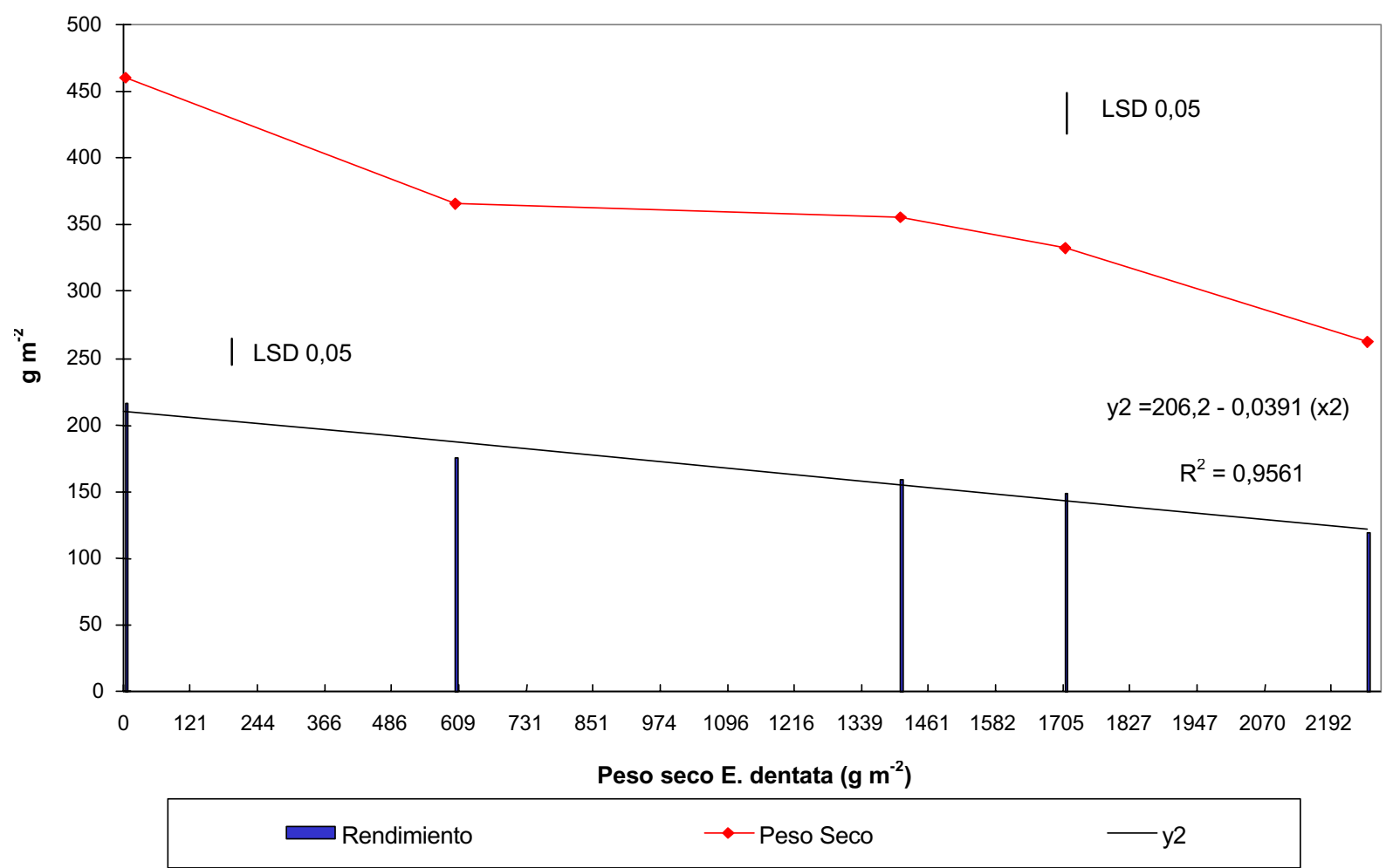

Figura 2 - Efecto de la biomasa de Euphorbia dentata sobre la biomasa seca y la productividad de soja. 
Asimismo, estudios previos de control químico de esta maleza han demostrado que la interferencia provocada por bajas densidades, 20 a 30 plantas $\mathrm{m}^{-2}$, remanentes de la aplicación de herbicidas que obtuvieron eficacia de control parcial (50 al 60\%), también manifiestan reducciones de rendimiento significativas en este cultivo (Juan, et al., 2000).

Las ecuaciones lineales determinadas en este estudio son probablemente una forma simplificada para estimar las pérdidas de productividad que provoca $E$. dentata en soja, sobre la base de las densidades mas frecuentemente encontradas en el área de estudio. No obstante en otros trabajos se ha reconocido un plateau que se comienza a manifestar por encima de las 160 plantas $\mathrm{m}^{-2} \mathrm{y}$ cuya importancia es relativa debido a que esas densidades actualmente representan menos del 2\% del área de distribución de la maleza (Juan et al., 1996).

De los componentes del rendimiento del cultivo, el numero de vainas por planta fue el más severamente afectado por la competencia registrándose diferencias significativas a partir de la mínima densidad estudiada, 20 plantas $\mathrm{m}^{-2}$, y llegando con la máxima densidad a una reducción del 40\% respecto del tratamiento sin maleza (Tabla 1).

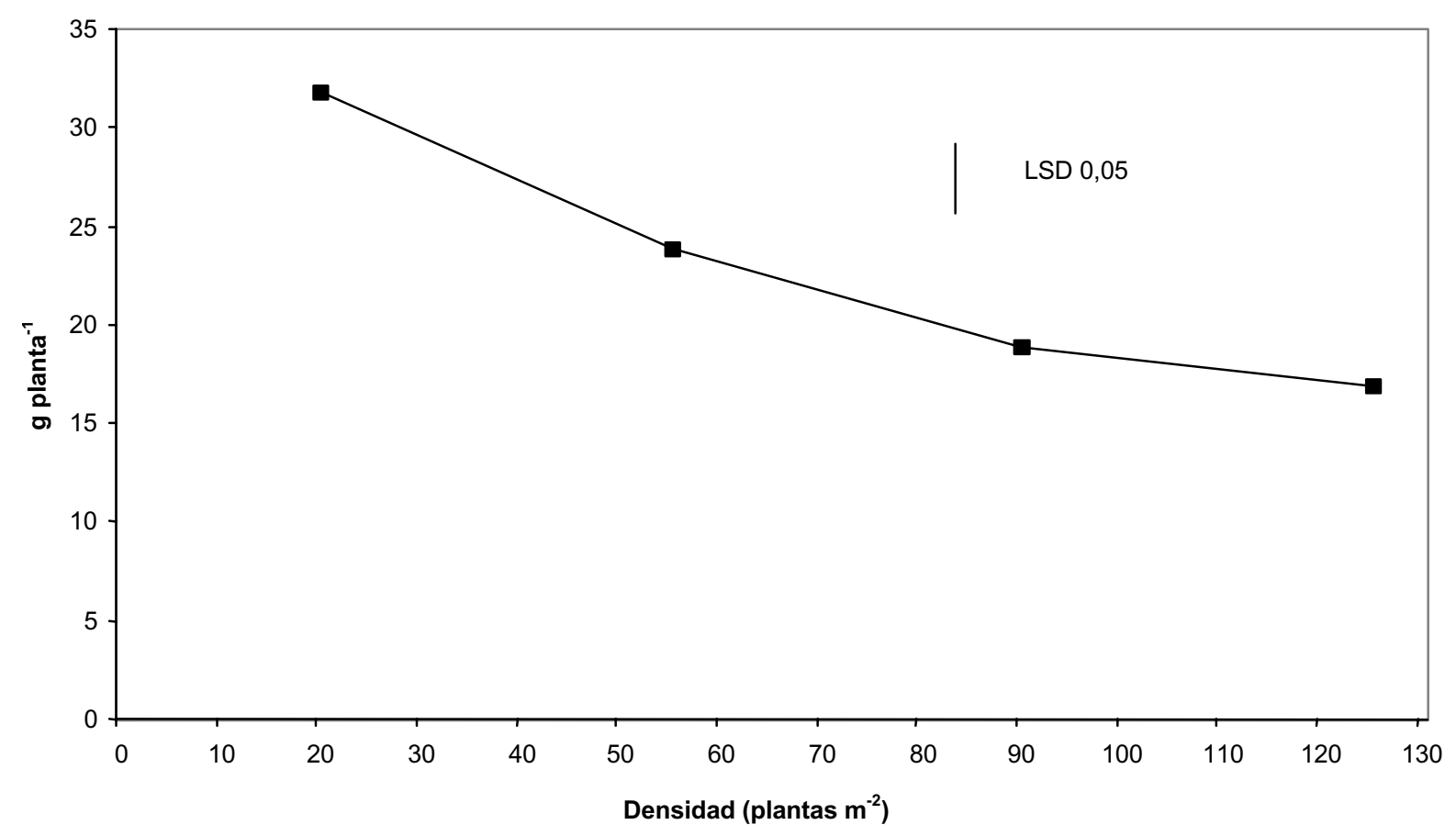

Figura 3 - Efecto de densidad de Euphorbia dentata sobre el peso seco por planta de la maleza.

Tabla 1 - Influencia de la densidad de Euphorbia dentata sobre los componentes del rendimiento de soja

\begin{tabular}{|c|c|c|c|}
\hline Densidad $\left(\right.$ plantas $\left.^{-2}\right)$ & Vainas planta $^{-1}$ & Semillas Vaina $^{-1}$ & Peso 1.000 semillas $(\mathrm{g})$ \\
\hline 0 & 20,2 & 2,18 & 159,6 \\
20 & 17,0 & 2,16 & 155,8 \\
55 & 15,2 & 2,09 & 149,2 \\
90 & 14,6 & 2,08 & 148,4 \\
125 & 12,3 & 2,04 & 144,6 \\
\hline LSD $(0,05)$ & 1,49 & 0,08 & 4,59 \\
\hline
\end{tabular}


El número de granos por vaina y el peso de 1000 semillas fueron significativamente afectados a partir de densidades relativamente altas de E. dentata, 55 plantas $\mathrm{m}^{-2}$, y con la mayor densidad sufrieron disminuciones del orden del $6,5 \%$ y $10 \%$ con respecto al testigo (Tabla 1).

Altos niveles de correlación fueron determinados entre la productividad del cultivo, el número de vainas por planta y el peso de 1.000 semillas ( 98 y $85 \%$ respectivamente).

\section{LITERATURA CITADA}

COBLE, H. D. Using economic thresholds for weeds in soybeans. En: CONFERENCIA MUNDIAL DE INVESTIGACION EN SOJA, 4, 1989, Buenos Aires. Proceedings... Buenos Aires: 1989. p. 1607-1612.

COUSENS, R. A simple model relating yield loss to weed density. Ann. Appl. Biol., v. 107, p. 239-252, 1985.

DEW, D. A. An index of competition for estimating crop loss due to weeds. Can. J. Plant Sci., v. 52, p. 921-927, 1972.

GIORDA, L. M.; BAIGORRI, H. E. J. (Eds.) El cultivo de la soja en Argentina. Ed. INTA: Centro Regional Córdoba, 1997. 448 p.

HARGER, T. R.; NESTER, P. R. Wild poinsettia: a major soybean weed. Louisiana Agric., v. 23, p. 4-7, 1980.

JUAN, V. F. et al. Estudios sobre lecherón (Euphorbia dentata Michaux) en la zona centro de la provincia de Buenos Aires. Planta Daninha, v. 14, n. 2, p. 102-109, 1996.
JUAN, V. F.; SAINT-ANDRE, H. M. Control de lecherón (Euphorbia dentata) en soja con imazethapyr aplicado en distintos estados fenológicos. En: CONGRESO

LATINOAMERICANO DE MALEZAS, 13., 1997, Buenos Aires, Argentina. Actas... Buenos Aires: ASAPROVE, 1997, v. 2, p. 128-133.

JUAN, V. F. et al. Control de Euphorbia dentata en soja con herbicidas post emergentes. R. Bras. de Herbic., v. 1, n. 2, p. 147-151, 2000.

NESTER, P. R.; HARGER, T. R.; Mc CORMICK, L. L. Weed watch-wild poinsettia. Weed Today, v. 10, p. 25, 1979.

PARODI, L. R. Ensayo fitogeográfico sobre el partido de Pergamino. R. Fac. Agron. y Veter. UBA, v. 8, n. 1, p. $218,1930$.

SWANTON, C. J.; WEISE S. F. Integrated weed management: the rationale and approach. Weed Techn., v. 5, p. 657-663, 1991.

WEAVER, S. E. Simulation of crop-weed competition: models and their applications. Phytoprotection, v. 77, p. 3-11, 1996.

WILLARD, T. S. et al. Interference of wild poinsettia (Euphorbia heteropylla) with soybean (Glicine max). Weed Techn., v. 8, p. 679-683, 1994.

WILSON, A. K. Euphorbia heterophylla: a review of distribution, importance and control. Trop. Pest Manag., v. 27 , p. 32-38, 1981.

ZIMDAHL, R. L. Weed-crop competition - A review. Corvallis, Oregon: International Plant Protection Center, 1980. p. 29. 